\title{
ATELE \\ Las territorialidades del turismo: el caso de los Pueblos Mágicos en México
}

\author{
A territorialidade do turismo: o caso de Pueblos Mágicos \\ no México
}

The territoriality of tourism: the case of Pueblos Mágicos in Mexico

Liliana López Levi

Universidad Autónoma Metropolitana. Unidad Xochimilco.

levi_lili@yahoo.com.mx

\begin{abstract}
Resumen
El programa Pueblos Mágicos es una iniciativa de la Secretaría de Turismo en México, para promover el desarrollo local. Desde el discurso, el turismo cultural se plantea como una estrategia para el crecimiento económico, el bienestar social y la sustentabilidad. Sin embargo, su implementación también conlleva prácticas de despojo, fragmentación y segregación. La puesta en valor del patrimonio produce múltiples territorialidades, que reflejan las dinámicas de desarrollo/despojo como dos caras de una misma moneda. Después de quince años de operación del programa, este artículo tiene como objetivo reflexionar sobre el impacto territorial, a partir del análisis de diversos casos de estudio. En una primera parte se exponen los referentes teóricos que nos permiten hablar de las múltiples territorialidades del turismo. Después se presentan los objetivos del programa, sus requisitos y reglas de operación para finalmente identificar los resultados del programa y contrastar el impacto territorial del programa Pueblos Mágicos con los discursos producidos en torno al turismo como estrategia de desarrollo.
\end{abstract}

Palabras clave: Turismo, territorio, desarrollo, Pueblos Mágicos

\section{Resumo}

O programa de "Pueblos Mágicos" é uma iniciativa do Secretaria de Turismo do México para promover o desenvolvimento local. A partir do discurso, o turismo cultural é planejado como uma estratégia para o crescimento econômico, o bemestar social e a sustentabilidade. Contudo, sua implementação também implica práticas de despojo, fragmentação e segregação. A valorização do patrimônio produz múltiplas territorialidades que se refletem nas dinâmicas do desenvolvimento/despojo, como as duas faces da mesma moeda. Após quinze anos de operação do programa, este artigo tem como objetivo refletir sobre o impacto territorial, a partir da análise de diversos estudos de caso. Numa primeira parte, 
apresentam-se os referenciais teóricos que nos permitem falar das múltiplas territorialidades do turismo. Posteriormente, apresentam-se os objetivos do programa, seus requisitos e regras de operação, para finalmente identificar os resultados do programa e comparar o impacto territorial do Programa "Pueblos Mágicos" com os discursos produzidos em torno do turismo como estratégia de desenvolvimento.

Palavras-chave: turismo, território, desenvolvimento, Pueblos Mágicos

\begin{abstract}
The program "Pueblos Magic" is an initiative of the Secretaria of Tourism of Mexico to promote local development. From the discourse, cultural tourism is planned as a strategy for economic growth, social well-being and sustainability. Although, your implementation also implies practices of dispossession, fragmentation and segregation. The valuation of heritage produces multiple territorialities that are reflected in the dynamics of development / dispossession, as the two sides of the same coin. The valuation of heritage produces multiple territorialities that are reflected in the dynamics of development / dispossession, as the two sides of the same coin. After fifteen years of operation of the program, the purpose of this article was reflecton about the territorial impact, based on the analysis of several case studies. In the first part, we present the theoretical references that allow us to speak of the multiple territorialities of tourism. Posteriorly, are presented the objectives of the program, its requirements and operating rules, to finally identify the results of the program and compare the territorial impact of the " Pueblos Mágicos" Program with the discourses produced around tourism as a development strategy.
\end{abstract}

Keywords: Tourism, territory, development, Pueblos Mágicos

\title{
Introducción
}

En el marco de la economía capitalista del siglo XXI, el turismo se ha situado como uno de los sectores económicos de mayor importancia. Se trata de una actividad desarrollada en espacios heterogéneos, que van desde las grandes ciudades hasta los paisajes naturales. Con ello, se revalora el patrimonio de los lugares modernos, los antiguos, las áreas naturales, las fiestas, las tradiciones, el arte; se desarrolla el mercado de segundas residencias, el ecoturismo, el turismo cultural, los parques de atracciones, los resorts con actividades recreativas y los hoteles diseñados para el descanso.

La derrama económica esperada por el aprovechamiento del patrimonio con fines culturales, recreativos y de ocio ha sido considerada como instrumento para impulsar crecimiento económico, el desarrollo social y la sostenibilidad ambiental. Todo ello ha implicado la transformación de los lugares; de su entorno natural, de las grandes urbes, del medio rural, de los sitios históricos y arqueológicos, así como las diversas expresiones culturales de la población local.

Con antecedentes en el siglo XX y con un gran impulso en el XXI, el turismo se sitúo como un importante nicho de mercado, una estrategia de desarrollo, tanto a nivel mundial, como nacional y local. En la página web de Organización Mundial de Turismo, se afirma que 
Durante décadas, el turismo ha experimentado un continuo crecimiento y una profunda diversificación, hasta convertirse en uno de los sectores económicos que crecen con mayor rapidez en el mundo. El turismo mundial guarda una estrecha relación con el desarrollo y se inscriben en él un número creciente de nuevos destinos. Esta dinámica ha convertido al turismo en un motor clave del progreso socioeconómico (OMT, 2017).

México no ha sido ajeno a esos discursos ni a las prácticas que derivan de estos. De acuerdo con dicha instancia, México ocupa el noveno lugar en llegadas de turistas internacionales (OMT, 2016). El discurso ha sido asumido al interior del país. El turismo ha sido planteado como uno de los ejes prioritarios para el desarrollo nacional, regional y local, en una forma que recuerda los tiempos en que se hablaba de industrialización. Se asume que impulsar el turismo genera empleos, derrama económica, bienestar social e incluso que es congruente con un programa de desarrollo sustentable.

Los discursos sobre el turismo orientan las políticas públicas y las iniciativas empresariales. Como resultado, se implementan programas concretos que al momento de ser instrumentados en el ámbito regional y local facilitan o dificultan ciertas prácticas socio-culturales que configuran múltiples territorialidades. Es decir, la puesta en valor del patrimonio con la idea de generar ganancias monetarias deriva en diversas formas de apropiación territorial.

En el año 2001, se puso en marcha un programa de desarrollo turístico en México, llamado Pueblos Mágicos. La iniciativa entra en concordancia con propuestas semejantes desarrolladas en otros países. En Francia, los pueblos se organizaron desde 1982 entorno a la asociación les plus beaux villages de France. Después, han aparecido otras iniciativas paralelas como la de Los pueblos más bonitos de España, i borghi più belli d'Italia, los pueblos con encanto en México y la Red de Pueblos Patrimonio de Colombia, entre otros ${ }^{l}$.

En particular, la presente investigación se avoca al caso de los Pueblos Mágicos, un programa promovido desde 2001 por la Secretaría de Turismo en México, con la idea de revalorar poblados significativos en el imaginario nacional y que tienen una riqueza patrimonial que puede ser aprovechada para el desarrollo local. En este sentido, se planteó como objetivo analizar las implicaciones territoriales que derivan de la implementación del programa Pueblos Mágicos en México.

El punto de partida son los discursos que justifican al programa como un instrumento de desarrollo local, para las comunidades con un patrimonio que puede ser aprovechado para el turismo. Posteriormente se exponen los requisitos y reglas de operación que deben cumplir las localidades que eligen esta vía, para finalmente

\footnotetext{
${ }^{1}$ Ver las páginas de dichas asociaciones http://www.les-plus-beaux-villages-de-france.org/fr/qui-sommesnous; $\quad$ http://www.lospueblosmasbonitosdeespana.org/; $\quad$ http://borghipiubelliditalia.it/borghi/; http://pueblospatrimoniodecolombia.org/
} 
identificar los resultados, es decir, las dinámicas de transformación de los lugares y las territorialidades resultantes.

El enfoque metodológico se sustenta en una aproximación cualitativa; en la cual se contrastan los discursos dominantes sobre el turismo y sus bondades, con las múltiples territorialidades que se manifiestan en lo local. Para identificar los resultados del programa y su impacto territorial se hicieron recorridos de campo, donde se realizó observación no participante, así como entrevistas libres y semiestructuradas con funcionarios, promotores turísticos, miembros de los Comités de Pueblos Mágicos, pobladores y turistas. La investigación documental incluyó la revisión de artículos de investigación, noticias de periódicos, folletos y promocionales turísticos, documentos gubernamentales y expedientes.

La información obtenida nos permitió conocer las motivaciones y expectativas de los actores locales, las estrategias para obtener la nominación, así como los resultados y las consecuencias socioculturales, económicas y ambientales de la implementación del programa.

En particular, hice recorridos en los pueblos de Huasca de Ocampo, Real del Monte y Mineral del Chico, en el Estado de Hidalgo; Huamantla, en el estado de Tlaxcala; Valle de Bravo y Metepec, en el Estado de México; Tequila, en el estado de Jalisco; Bacalar, en Quintana Roo; Dolores Hidalgo, en el estado de Guanajuato; Zacatlán y Chignahuapan, en el estado de Puebla. Sin embargo, los resultados de investigación que presento son concordantes con los hallazgos y conclusiones de un amplio equipo de investigadores, con quienes analizamos 60 casos de los 111 Pueblos Mágicos que hay actualmente en México².

\section{Las múltiples territorialidades del turismo}

La gran diversidad natural y cultural de México ha facilitado la utilización del patrimonio con fines de desarrollo económico a través del turismo. Lo anterior queda plasmado en discursos que se hacen patentes en documentos oficiales, folletos de promoción turística, comunicaciones en radio, televisión, internet, anuncios espectaculares, mensajes de los operadores turísticos, discursos de funcionarios públicos en distintos niveles y con filiaciones partidistas diversas.

En el Plan Nacional de Desarrollo 2013-2018 se establece que

El turismo es prioritario para el Gobierno de la República porque genera una importante derrama económica, impulsa el crecimiento sostenido y genera empleos que mejoran la calidad de vida de los mexicanos. El sector es estratégico porque fomenta el desarrollo regional equilibrado y sustentable, integra a los habitantes de las distintas localidades, especialmente a jóvenes y mujeres, a la vida productiva y fomenta un crecimiento justo y equitativo. La actividad turística es el gran vehículo para transitar

\footnotetext{
${ }^{2}$ La investigación se sustenta en un proyecto financiado por el Consejo Nacional de Ciencia y Tecnología en México, del cual fui responsable y que se desarrolló de noviembre de 2012 a noviembre de 2016.
} 
hacia un México más próspero e incluyente (Gobierno de la República, 2013: 12).

El Consejo de Promoción Turística en México, a través de su página web, afirma que "la importancia del turismo para la economía mexicana es indudable, sus beneficios no solo se reflejan en ser una industria que genera empleos y en ser un detonador del desarrollo local y regional, sino que además es factor de difusión de atractivos culturales y naturales."

Por su parte, la Secretaría de Turismo emite frecuentemente comunicados. Dos de los últimos encontrados, con fecha del 20 de febrero de 2017, afirman que:

El turismo se ha convertido en la nueva plataforma para el desarrollo social y económico del país, porque al impulsar un destino turístico se desarrolla también su alrededor, se genera progreso a través de vialidades, inversiones, servicios públicos y oportunidades de empleo (SECTUR, 2017).

El producto Interno Bruto (PIB) turístico registró un crecimiento anual de 4.3 por ciento durante el tercer trimestre de 2016, más del doble que la economía nacional que en el mismo lapso creció 2.0 por ciento, informó la secretaría de Turismo (Sectur) del Gobierno de la República (...) Asimismo, indicó que el consumo turístico en el país registró un crecimiento de 6.4 por ciento de enero a septiembre de 2016. (...)Precisó que el consumo de los turistas nacionales aumentó 3.3 por ciento, mientras que el consumo de los turistas internacionales creció 24 por ciento en el mismo periodo (SECTUR, 2017).

Estos discursos, como muchos otros más, muestran un panorama optimista, que conlleva posibilidades de crecimiento económico, bienestar social, alternativas a la crisis y resolución de problemas tales como el desempleo y la pobreza. Aunque muchos académicos han cuestionado la manera en que se concretan las expectativas, los funcionarios públicos, sin distinguir matices, lo ven como una buena opción para el progreso.

En la Dialéctica de la ilustración, Adorno y Horkheimer (2006) señalan que la aparente oposición integrada entre la barbarie y la civilización, no se refiere a dos extremos de un proceso o a situaciones excluyentes, sino a dos caras de la misma moneda. Bajo esta misma lógica considero pertinente analizar la puesta en valor del patrimonio y las consecuencias de la implementación de un programa de turismo, mediante la dupla desarrollo/despojo, que se expresa a través de la configuración de diversas territorialidades.

Para el caso de los Pueblos Mágicos, mientras que las políticas públicas y los discursos que las secundan prometen desarrollo local y sustentabilidad, el análisis de los pueblos muestra un panorama distinto, donde surgen espacios desiguales, injustos, segregados, subvalorados y ambientalmente deteriorados (Valenzuela et al, 2014; 
López Levi et al, 2015; 2017; Hernández Mar, 2015; Méndez, 2016; Alvarado et al, 2016).

La puesta en valor del patrimonio plantea recuperar el paisaje, elementos naturales, tradiciones religiosas, sitios históricos, costumbres, tradiciones y productos agrícolas o artesanales para atraer visitantes y reactivar la economía. Se trata de un programa instrumentado en tiempos del neoliberalismo y de la globalización, con una sociedad manipulada por la lógica del consumo, en tiempos de incertidumbre, con poca credibilidad en el gobierno, inseguridad, pobreza, malas condiciones laborales y abandono del campo, entre otras problemáticas.

Con base en autores tales como Haesbert (2011, 2013), Giménez (1999) y Rodríguez (2015) podemos decir que la apropiación material, social y simbólica del espacio confligura territorialidades. Éstas se producen a partir de diversos mecanismos, que implican poseción, intervención o gestión de los lugares. Es una relación de poder que se establece con la tierra, con el suelo y con sus habitantes; una que se da independientemente de la aceptación o rechazo de los pobladores. Cabe aclarar que no toda apropiación es material; las territorialidades también tienen un componente simbòlico o emocional que configura los vinculos afectivos con porciones de la superficie de la tierra.

Si retomamos la crítica anarquista al capitalismo, podemos afirmar que en la sociedad moderna y actual hay tres fuerzas dominantes: el Estado, la Iglesia y el capital (Quesada, 2016), a partir de las cuales podemos derivar la jerarquía de territorialidades que se concretan en el ámbito local.

Las formas de apropiación se construyen a partir de los "vínculos de pertenencia y apego y desde donde toman forma los proyectos de vida, para lo cual ejercen o se enfrentan a relaciones de dominio y control" (Rodríguez Wallenius, 2015: 31). El resultado son las distintas territorialidades que suelen estructurar a la vida humana en términos culturales, demográficos, políticos, económicos y ambientales. Algunas se manifiestan con procesos de exclusión, como los que señalan autores como Castellanos y Machuca (2008); Hernández (2008), González (2010) y Duarte (2015) o en las formas de apropiación de los recursos por parte de los grupos de poder económico y político (Daltabuit et al, 2007).

\section{El programa Pueblos Mágicos}

Un Pueblo Mágico es, de acuerdo con la Secretaría de Turismo (SECTUR), "una localidad que tiene atributos simbólicos, leyendas, historia, hechos trascendentes, cotidianidad, en fin MAGIA que emana en cada una de sus manifestaciones socio culturales, y que significan hoy día una gran oportunidad para el aprovechamiento turístico".

El reconocimiento del valor patrimonial y simbólico de dichas localidades, por medio de un programa de fomento al turismo, se estableció con la intención de contribuir "a revalorar a un conjunto de poblaciones del país que siempre han estado en el imaginario colectivo de la nación en su conjunto y que representan alternativas 
frescas y diferentes para los visitantes nacionales y extranjeros. Más que un rescate, es un reconocimiento a quienes habitan esos hermosos lugares de la geografía mexicana y han sabido guardar para todos, la riqueza cultural e histórica que encierran" (SECTUR, 2012).

El programa inició formalmente en 2001, cuando el sistema político mexicano estaba en un periodo de transición, después de varias décadas de tener en el gobierno a un partido hegemónico. En ese tiempo se hablaba de alternancia política, de transición a la democracia y se asumía que la participación ciudadana sería mayor y su voluntad más respetada. Aunque la propuesta turística se implementó con un nuevo partido en el poder, varios funcionarios locales apuntan que fue concebido y planeado en el sexenio anterior $^{3}$.

El primer pueblo en ser nombrado Pueblos Mágicos fue Huasca de Ocampo en 2001; al año siguiente, Mexcaltitlán, Tepoztlán y Real del Catorce. Después entraron, Comala, Cuetzalan, Dolores Hidalgo, Izamal, Pátzcuaro, San Miguel de Allende, Tapalpa Taxco y Tepozotlán. Y así, a lo largo de doce años de gobiernos panistas ${ }^{4}$ fueron incorporándose uno a uno hasta llegar a 83, en 2012, a pesar de que en algún momento la titular de la Secretaría de Turismo declaró que la meta era llegar a 52, uno para cada fin de semana. En el proceso, también hubo localidades que salieron de programa. Tal fue el caso de Tepoztlán, Papantla, Mexcaltitlán y San Miguel de Allende. Los dos primeros recuperaron su nombramiento y los otros dos no. El segundo, San Miguel de Allende dejó el programa como consecuencia de su nombramiento de Patrimonio de la Humanidad por la UNESCO. En palabras de un entrevistado, miembro de uno de los comités de Pueblo Mágico "tuvieron que dejar la moto para tener el coche".

En el año 2012, antes de finalizar el sexenio, se nombraron 34 pueblos. La premura por incorporar nuevas localidades antes de terminar la gestión del presidente Felipe Calderón llevó a la conformación de expedientes apresurados y a nominaciones posteriormente cuestionadas. Después, en el primer trienio del gobierno de Enrique Peña Nieto, el programa entró en un proceso de evaluación. Algunos pueblos que no cumplían con las reglas (como tener un expediente y un comité) tuvieron que arreglar su situación ante la amenaza de perder el nombramiento; otros prepararon sus candidaturas ante una nueva convocatoria y finalmente, en septiembre de 2015 se anunció la incorporación de 28 nuevos pueblos al programa, con lo cual se alcanzó un número de 111 Pueblos Mágicos, muchos más de los programados en un inicio. A la fecha corresponde al número actual, aunque se habla de incorporar más pueblos el próximo año.

\footnotetext{
${ }^{3}$ El programa inició su implementación durante la presidencia de Vicente Fox (Partido Acción Nacional, 2000-2006), con la empresaria Leticia Navarro Sánchez en la Secretaría de Turismo (diciembre 2000 a julio 2003).

${ }^{4}$ En las gestiones de Rodolfo Elizondo Torres (agosto 2003 a marzo de 2010) y Gloria Guevara Manzo (marzo de 2010 a noviembre de 2012) al frente de la Secretaría de Turismo.
} 


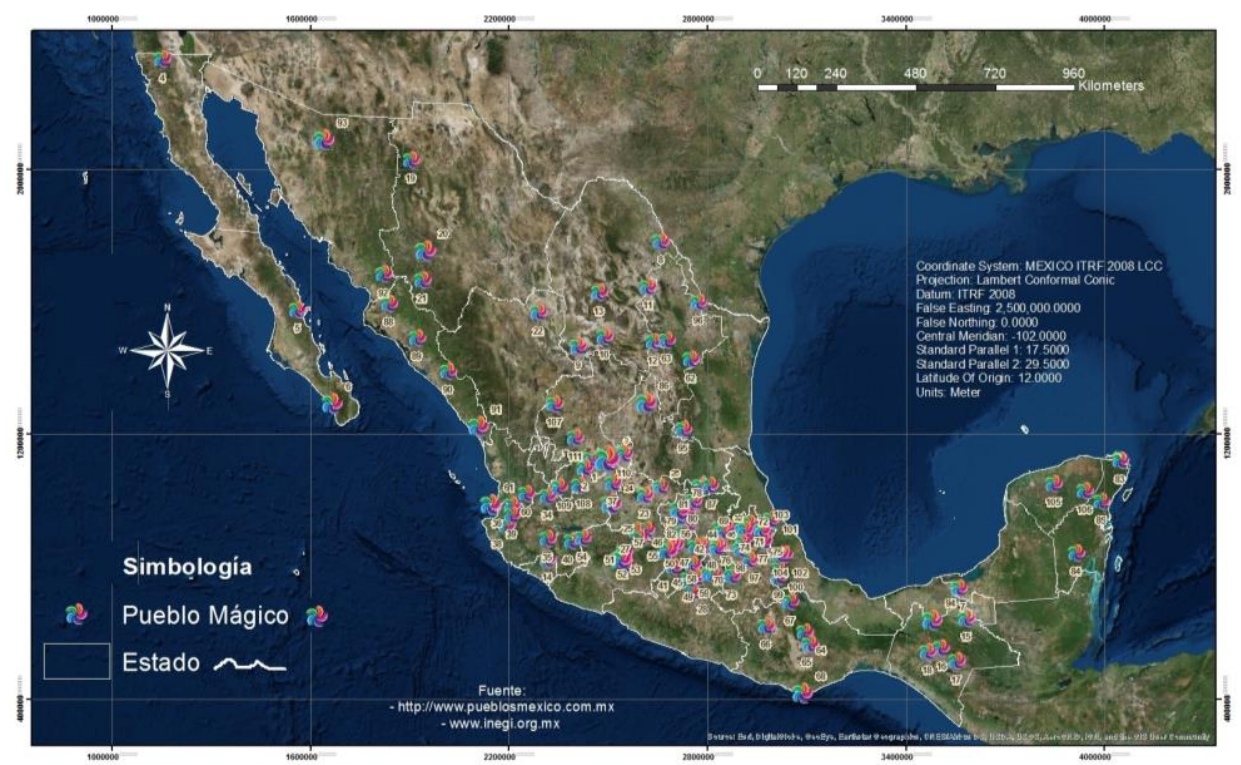

Figura 01. Pueblos Mágicos, México 2017.

Elaboración: Hayde Bustamante 2017

Tabla 01. Pueblos Mágicos de México

\begin{tabular}{|c|c|c|c|c|c|}
\hline \multicolumn{2}{|c|}{ PUEBLO MÁGICO } & ESTADO & \multicolumn{2}{c|}{ PUEBLO MÁGICO } & ESTADO \\
\hline 1 & San José de Gracia & AGUASCALIENTES & 29 & Huichapan & HIDALGO \\
\hline 2 & Calvillo & AGUASCALIENTES & 30 & Huasca de Ocampo & HIDALGO \\
\hline 3 & Real de Asientos & AGUASCALIENTES & 31 & Mineral del Chico & HIDALGO \\
\hline 4 & Tecate & BAJA CALIFORNIA & 32 & Real de Monte & HIDALGO \\
\hline 5 & Loreto & $\begin{array}{c}\text { BAJA CALIFORNIA } \\
\text { SUR }\end{array}$ & 33 & Tecozautla & HIDALGO \\
\hline 6 & Todos Santos & $\begin{array}{c}\text { BAJA CALIFORNIA } \\
\text { SUR }\end{array}$ & 34 & Tequila & JALISCO \\
\hline 7 & Palizada & CAMPECHE & 35 & Tapalpa & JALISCO \\
\hline 8 & Guerrero & COAHUILA & 36 & San Sebastián del & JALste \\
\hline 9 & Viesca & COAHUILA & 37 & Lagos de Moreno & JALISCO \\
\hline 10 & Parras de la Fuente & COAHUILA & 38 & Talpa de Allende & JALISCO \\
\hline 11 & Candela & COAHUILA & 39 & Mascota & JALISCO \\
\hline 12 & Arteaga & COAHUILA & 40 & Mazamitla & JALISCO \\
\hline
\end{tabular}




\begin{tabular}{|c|c|c|c|c|c|}
\hline \multicolumn{2}{|r|}{ PUEBLO MÁGICO } & \multirow{2}{*}{$\begin{array}{c}\text { ESTADO } \\
\text { COAHUILA }\end{array}$} & \multicolumn{2}{|c|}{ PUEBLO MÁGICO } & \multirow{2}{*}{$\begin{array}{c}\text { ESTADO } \\
\text { EDO. MEX. }\end{array}$} \\
\hline 13 & Cuatro Ciénegas & & 41 & Valle de Bravo & \\
\hline 14 & Comala & COLIMA & 42 & Aculco & EDO. MEX. \\
\hline 15 & Palenque & CHIAPAS & 43 & Tepotzotlán & EDO. MEX. \\
\hline 16 & $\begin{array}{c}\text { San Cristobal de las } \\
\text { Casas }\end{array}$ & CHIAPAS & 44 & Villa del carbón & EDO. MEX. \\
\hline 17 & Comitán & CHIAPAS & 45 & Metepec & EDO. MEX. \\
\hline 18 & Chiapa de Corzo & CHIAPAS & 46 & El Oro & EDO. MEX. \\
\hline 19 & Casas Grandes & CHIHUAHUA & 47 & Malinalco & EDO. MEX. \\
\hline 20 & Creel & CHIHUAHUA & 48 & San Juan Teotihuacán & EDO. MEX. \\
\hline 21 & Batopilas & CHIHUAHUA & 48 & $\begin{array}{c}\text { San Martín de las } \\
\text { Pirámides } \\
\end{array}$ & EDO. MEX. \\
\hline 22 & Mapimi & DURANGO & 49 & Ixtapan de la Sal & EDO. MEX. \\
\hline 23 & Dolores Hidalgo & GUANAJUATO & 50 & Tlalpujahua & MICHOACAN \\
\hline 24 & Jalpa & GUANAJUATO & 51 & Tacámbaro & MICHOACAN \\
\hline 25 & Salvatierra & GUANAJUATO & 52 & Pátzcuaro & MICHOACAN \\
\hline 26 & Mineral de Pozos & GUANAJUATO & 53 & Tzintzuntzan & MICHOACAN \\
\hline 27 & Yuriria & GUANAJUATO & 54 & Jiquilpan & MICHOACAN \\
\hline 28 & Taxco & GUERRERO & 55 & Cuitzeo & MICHOACAN \\
\hline 56 & Mineral de Angangueo & MICHOACAN & 84 & Bacalar & Q. ROO \\
\hline 57 & Santa Clara del Cobre & MICHOACAN & 85 & Tulum & Q. ROO \\
\hline 58 & Tepoztlán & MORELOS & 86 & Real de Catorce & S.L.P. \\
\hline 59 & Tlayacapan & MORELOS & 87 & Xilitla & S.L.P. \\
\hline 60 & Jala & NAYARIT & 88 & El Fuerte & SINALOA \\
\hline 61 & Sayulita & NAYARIT & 89 & Mocorito & SINALOA \\
\hline 62 & Linares & NUEVO LEÓN & 90 & Cosalá & SINALOA \\
\hline 63 & Santiago & NUEVO LEÓN & 91 & El Rosario & SINALOA \\
\hline 64 & Capulálpam de Méndez & OAXACA & 92 & Álamos & SONORA \\
\hline 65 & San Pablo Villa Mitla & OAXACA & 93 & Magdalena de Kino & SONORA \\
\hline 66 & $\begin{array}{c}\text { San Pedro y San Pablo } \\
\text { Teposcolula }\end{array}$ & OAXACA & 94 & Tapijulapa & TABASCO \\
\hline 67 & Huautla de Jiménez & OAXACA & 95 & Tula & TAMAULIPAS \\
\hline 68 & Mazunte & OAXACA & 96 & Mier & TAMAULIPAS \\
\hline
\end{tabular}




\begin{tabular}{|c|c|c|c|c|c|}
\hline \multicolumn{2}{|c|}{ PUEBLO MÁGICO } & ESTADO & \multicolumn{2}{c|}{ PUEBLO MÁGICO } & ESTADO \\
\hline 69 & Pahuatlán & PUEBLA & 97 & Huamantla & TLAXCALA \\
\hline 70 & San Pedro Cholula & PUEBLA & 98 & Tlaxco & TLAXCALA \\
\hline 71 & Xicotepec & PUEBLA & 99 & Orizaba & VERACRUZ \\
\hline 72 & Zacatlán & PUEBLA & 100 & Coscomatepec & VERACRUZ \\
\hline 73 & Atlixco & PUEBLA & 101 & Papantla & VERACRUZ \\
\hline 74 & Huauchinango & PUEBLA & 102 & Coatepec & VERACRUZ \\
\hline 75 & Cuetzalan del Progreso & PUEBLA & 103 & Zozocolco & VERACRUZ \\
\hline 76 & Chignahuapan & PUEBLA & 104 & Xico & VERACRUZ \\
\hline 77 & Tlatlauquitepec & PUEBLA & 105 & Izamal & YUCATAN \\
\hline 78 & Bernal & QUERÉTARO & 106 & Valladolid & YUCATAN \\
\hline 79 & Cadereyta de Montes & QUERÉTARO & 107 & Sombrerete & ZACATECAS \\
\hline 80 & San Joaquín & QUERÉTARO & 108 & Nochistlán & ZACATECAS \\
\hline 81 & Jalpan de Serra & QUERÉTARO & 109 & $\begin{array}{c}\text { Teúl de González } \\
\text { Ortega }\end{array}$ & ZACATECAS \\
\hline 82 & Tequisquiapan & QUERÉTARO & 110 & Pino & ZACATECAS \\
\hline 83 & Isla Mujeres & Q. ROO & 111 & $\begin{array}{c}\text { Jeréz de García } \\
\text { Salinas }\end{array}$ & ZACATECAS \\
\hline
\end{tabular}

Fuera del programa han quedado muchas localidades de gran riqueza histórica, natural y cultural, pero que no se han propuesto o no han logrado reunir los requisitos ni gestionar su incorporación. Hay otras tantas que están trabajando con la ilusión de abrirse nuevas alternativas de derrama económica ${ }^{5}$, bienestar social y desarrollo sustentable.

Cabe destacar que la proporción de pueblos que pertenecen al programa es mucho mayor en el centro del país, lo que algunos investigadores asumen como consecuencia de la distribución real de los poderes políticos y de las élites. Con ello se da cuenta que no se trata exclusivamente de "magia" o riqueza patrimonial, las redes económicas y políticas, así como la capacidad de armar un expediente y gestionarlo son aún más importantes.

\section{Requisitos y reglas de operación}

El punto de partida para declarar a un pueblo mágico es la posibilidad de aprovechar su singularidad, de poner en valor el patrimonio y estructurar una oferta

\footnotetext{
${ }^{5}$ Desde las esferas del poder se habla de crecimiento económico, en cambio los actores locales suelen usar más frecuentemente el término de derrama económica.
} 
turística. Como se ha mencionado en párrafos anteriores, el objetivo a lograr es el crecimiento económico, el bienestar social y el desarrollo sustentable. Con base en lo anterior, en los discursos del programa se ofrece "mayor gasto en beneficio de la comunidad receptora", "creación y/o modernización de los negocios turísticos locales" y que el turismo sea una actividad redituable, que sea una herramienta para el desarrollo sustentable". En tiempos de crisis económica, política y social, es una forma de levantar ánimos y generar nuevas expectativas.

En las Reglas de Operación del programa se establece como objetivos:

- Estructurar una oferta turística complementaria y diversificada hacía el interior del país, basada fundamentalmente en los atributos histórico - culturales de localidades singulares.

- Aprovechar la singularidad de las localidades para la generación de productos turísticos basados en las diferentes expresiones de la cultura local; artesanías, festividades, gastronomía, y tradiciones, entre otras.

- Aprovechar la singularidad de las localidades para la generación de otros productos turísticos tales como la aventura y el deporte extremo, el ecoturismo, la pesca deportiva, y otros que signifiquen un alto grado de atractividad dentro del territorio de la localidad participante.

- Poner en valor, consolidar y/o reforzar los atractivos de las localidades con potencial y atractividad turística, fomentando así flujos turísticos que generen:

- Mayor gasto en beneficio de la comunidad receptora (artesanías, gastronomía, amenidades y el comercio en general), así como,

- La creación y/o modernización de los negocios turísticos locales.

- Que el turismo local se constituya como una herramienta del desarrollo sustentable de las localidades incorporadas al programa, así como en un programa de apoyo a la gestión municipal.

- Que las comunidades receptoras de las localidades participantes aprovechen y se beneficie del turismo como actividad redituable como opción de negocio, de trabajo y de forma de vida (SECTUR, 2001).

Sin embargo, tal y como se establece después en las mismas Reglas de Operación de la Secretaría de Turismo, la singularidad de la localidad y sus atributos histórico-culturales y naturales, no son suficientes para gestionar el turismo y lograr 
una rentabilidad económica; por lo que el nombramiento debe ir acompañado de diversas certificaciones de calidad que otorga la misma Secretaría de Turismo. Entre estos destacan Punto Limpio, Distintivo H, Moderniza y SuperHost. Estas certificaciones se otorgan a las micro, pequeñas y medianas empresas del sector por implementar la metodología desarrollada por SECTUR, para estimular e incrementar índices de rentabilidad y competitividad, así como cumplir con los estándares de manejo higiénico de alimentos y por seguir los programas de capacitación. Asimismo, se promueve que se ofrezcan actividades, itinerarios, rutas turísticas e infraestructura para dar servicio a los clientes potenciales.

Las Reglas de Operación del Programa de Pueblos Mágicos (SECTUR, 2001) establecen ocho requisitos para la incorporación y permanencia dentro del programa:

1. Involucramiento de la Sociedad y de las Autoridades Locales.

2. Instrumentos de Planeación y Regulación.

3. Impulso al Desarrollo Municipal.

4. Oferta de Atractivos y Servicios.

5. Valor Singular "La Magia de la Localidad".

6. Condiciones y Espacios Territoriales.

7. Impacto del Turismo en la Localidad y Área de Influencia.

8. Desarrollo de Capacidades Locales

Los puntos anteriores han sido la guía para organizar el expediente y aunque en el papel, el programa contempla que los pobladores locales se involucren y participen, como ciudadanos; en la práctica ello se reduce a la recaudación de firmas y al involucramiento únicamente de aquellos que tienen interés en el sector turístico. Los requisitos también asumen una adecuada relación con las autoridades, una coparticipación entre el sector público y el privado, la existencia de planes y programas de ordenamiento territorial y de planeación. Muchos de los cuales son buenas intenciones que frecuentemente quedan solo como requisito.

De acuerdo con algunos entrevistados que vivieron el proceso, la iniciativa de pertenecer al programa, muchas veces viene de las autoridades estatales. Si bien, la solicitud debe estar avalada por la población local, en el entendido de que se involucra a la sociedad civil en la determinación, en algunos casos incluso fueron nombrados sin que la población supiera del proceso. Tal fue el caso de los pueblos de Dolores Hidalgo, Guanajuato; Jalpa, Guanajuato; Valle de Bravo, Estado de México y Nochistlán, Zacatecas Ellos suelen expresarlo en términos de que cuando se enteraron, ya eran pueblo mágico. "Nos lo dieron de dedazo" dice la presidenta del Comité de Pueblo Mágico de Dolores Hidalgo.

En otros casos hubo un grupo local o el mismo ayuntamiento que decidió o aceptó hacer la solicitud de incorporación y entonces tuvieron que conformar el Comité Pro Pueblo Mágico, integrar el expediente o contratar a alguien que lo hiciera. Hay empresas que cobran fuertes sumas de dinero por ello. 
A lo largo de los años y en las diversas localidades ha habido Comité Pro Pueblo Mágico que en verdad funcionan y se integran y otros que están de nombre y son solo simulación. Sus integrantes no se reúnen periódicamente ni se involucran en las decisiones con respecto al Pueblo Mágico. También hay muchos casos en los cuales sus miembros son activos y trabajan bien en equipo, por ejemplo en Zacatlán, Puebla. Es importante destacar que se trata de un puesto honorario que implica responsabilidades, más no remuneración, por lo que el trabajo depende del entusiasmo de sus miembros.

Entre las tareas está la de reflexionar sobre las características locales que justifican la singularidad del lugar y su valor patrimonial. Para ello se crea o se enaltece un producto turístico (algún festival, artesanía, fiesta patronal, gastronomía, etc). Dicho producto debe sustentarse con servicios para los visitantes (hoteles, restaurantes), mismos que pasan por un proceso de certificación.

\section{Las territorialidades del Programa}

En el discurso, el programa Pueblos Mágicos ofrece ser un instrumento para el desarrollo local, a través de la valoración de la magia, de la población, del patrimonio y del territorio, por medio del turismo cultural. En este sentido, plantea la posibilidad de beneficiar a pequeñas localidades que habían quedado al margen del sector turístico, mismo que durante el siglo XX benefició primordialmente a los destinos de sol y playa, arqueológicos y algunas ciudades coloniales.

Pueblos Mágicos abre la oportunidad de recuperar también el patrimonio cultural, festivo, gastronómico, natural, artístico, histórico e incluso la capacidad de propuesta y gestión de algunos grupos locales. Sin embargo, el programa también alberga a los poblados con una gran trayectoria turística y que sirven de modelo a seguir.

En términos generales y con base en los resultados del trabajo de campo desarrollado a lo largo de poco más de 5 años en diversas localidades del país, hemos registrado que el programa es ampliamente aceptado por parte de muchos emprendedores locales, de los operadores turísticos y de los funcionarios públicos. Sin embargo, gran parte de la población local se encuentra al margen de las iniciativas y los beneficios ofrecidos por el programa. De tal manera que mientras algunos se expresan orgullosos de sus logros, otros ven sus expectativas incumplidas y muchos se sienten ajenos a las bondades del programa. Es importante destacar que la valoración de las virtudes y problemas de la turistificación del patrimonio varían de acuerdo a la posición y la experiencia de los distintos actores sociales entrevistados.

En tiempos del neoliberalismo, el Estado deja de ser el productor del espacio social y se convierte en el promotor de las dinámicas económicas. Los procesos derivados de la implementación del programa configuran múltiples territorialidades, en las cuales, destaca el actuar de las élites locales, como fuerza dominante.

Las territorialidades dominantes ponen al turismo, como actividad económica, en el centro de la vida cotidiana, del desarrollo local, humano y sustentable. Esto quiere 
decir que las actividades afines a los servicios turísticos y a la puesta en valor del patrimonio serán las que tengan prioridad sobre la ocupación del espacio y sobre el trazado del destino de la localidad.

Uno de las consecuencias más visibles del programa es la intervención en la imagen urbana. Aunque el programa contempla recursos para la imagen urbana, muchas localidades invierten previamente en infraestructura. Se pintan y arreglan fachadas, se ocultan los cables, se cambia el pavimento, se quitan árboles, se rehabilitan espacios públicos, en otras palabras se embellece el lugar para agradar al turista. ¿Quién decide los colores adecuados, el estilo arquitectónico y la obra pública a realizar? Los funcionarios locales y las instituciones nacionales o regionales que regulan el patrimonio (Instituto Nacional de Antropología e História y la Junta de Monumentos de Zacatecas, por mencionar dos ejemplos).

Aunque en general, los actores locales coinciden en la idea de invertir en la fisonomía de la localidad, no todos coinciden en gustos. Es dominante la visión en la cual se busca emular un pasado colonial, que a veces lleva a la simulación, más no a la restauración de los inmuebles. En la investigación también registramos como idea dominante, el imaginario del pueblito mexicano con casas blancas con guardapolvo rojo, techos de dos aguas con tejas y calles empedradas. Un paisaje que se reproduce como auténtico en el centro del país y que preocupa a los pueblos periféricos que no cumplen con la imagen. Tal es el caso de Bacalar, en Quintana Roo, o de Tecate, en Baja California.

Una de las críticas registradas en varias localidades es que el arreglo de la imagen urbana se reduce la plaza central y su entorno. "A 150 metros se acaba la magia" dice uno de los entrevistados. Algo semejante registra José de Jesús Hernández (2009: 56) en el pueblo de Tequila, Jalisco, cuando habla de los problemas de la localidad y afirma que contrastan "con un centro mágico que no sólo es cosméticamente más visible y vistoso, sino que permite ocultar las condiciones reales del pueblo tradicional".

La idea de mejorar la imagen también ha llevado a desplazar actividades y personas que constituían parte importante del paisaje y de la vida social de una comunidad. Ejemplo de esto ha sido el desplazamiento de las ferias y mercados, del centro a la periferia o el desalojo de los vendedores ambulantes de las plazas. Algunos, dice un entrevistado, son personas que venden ahí desde hace tres o cuatro generaciones y que los habitantes locales consideran como lo típico del lugar. Este tipo de acciones transgreden las territorialidades de los pobladores.

En el análisis del pueblo mágico de Cuitzeo, Michoacán, Esperanza Duarte denuncia un imaginario impuesto que "lleva implícito determinados valores y símbolos, como la estetización del patrimonio edificado y del espacio público, la limpieza, la seguridad y la búsqueda de negocios bien" y que tiene como consecuencia la exclusión de la población local (Duarte, 2015: 172). La autora transcribe algunas frases de las entrevistas hechas por ella y que son muy elocuentes de las dinámicas de segregación, despojo y desplazamiento. "no dejan que los niños vayan y corran (...) no hay la confianza que había antes" (Duarte, 2015:175). "Las manifestaciones de rechazo hacia lo local son más marcadas en cada ocasión que se realizan fiestas o eventos 
políticos exclusivos en el ex convento agustino y la plaza de Cuitzeo, ya que por seguridad de los invitados, se cierran las calles aledañas al centro y desalojan a los vecinos de las calles" (Duarte, 2015:176).

Ramírez et al (2017) en su estudio sobre Malinalco, otro de los pueblos mágicos del Estado de México, exponen diversas situaciones como las de un inversionista que compró una hacienda y cercó la capilla a la que ya no pudieron acceder los locales; la de un hombre de negocios que quiso despojar a un barrio de su manantial o la de un empresario que compró su casa de descanso frente a una capilla y se enojaba cuando había fiesta patronal porque los cohetes no lo dejaban descansar.

Para el caso de Tequila, Jalisco, Hernández (2009: 56) muestra que, a pesar de las promesas del programa, en la localidad siguen padeciendo problemas relacionados con el acceso al agua, con la basura, el mantenimiento de la infraestructura urbana, la criminalidad y corrupción. En otro artículo, el mismo autor, junto con Elizabeth Hernández afirma que en Tequila "hay más trabajo pero no mejores condiciones de vida, tampoco participación social. Interesa la fuerza de trabajo pero no los trabajadores en sí” (Hernández y Hernández, 2010: 275).

Por su parte, Lucía González (2010) hace un registro visual de los procesos de exclusión en Tequila, a través de imágenes con letreros de los productores de agave donde plasman que "aquí comienza la Ruta del Tequila para el paisaje agavero, patrimonio de la humanidad y zona de desastre y miseria de los agaveros" y que "éste es el pueblo mágico para la industria y pretexto para el gobierno para ayuda de unos cuantos... pero desgracia y pobreza de los agaveros."

El caso de Tequila sirve también para dar cuenta de las territorialidades de la agricultura. En el paisaje agavero, la siembra del insumo de la bebida ha desplazado otros cultivos, así como otros usos del suelo rural. Algo semejante ocurre con otros pueblos, como Zacatlán de las Manzanas, en Puebla, que dejan de lado su producción agrícola para centrarse en el turismo.

Con respecto al discurso del desarrollo sustentable, en el caso de los pueblos mágicos podemos afirmar que la naturaleza se convierte en negocio. Si bien se habla de la sustentabilidad ambiental, ésta se confunde con el uso del paisaje y sus elementos biológicos y geomorfológicos para atraer al turista. Entonces, en medio de parques naturales, como en el caso del pueblo mágico Mineral del Chico en Hidalgo o Valle de Bravo en el Estado de México se ofrecen tirolesas, cuatrimotos, lugares para escalar a rappel.

Por otro lado, el programa de Pueblos Mágicos también ha dado la oportunidad de que aflore la herencia colonial y los episodios de la historia donde la Iglesia resultó vencida. Son numerosos los pueblos que tienen como centro de su valor patrimonial a iglesias y conventos. Entre otros se puede mencionar a Izamal, en Yucatán y a Tepozotlán en el Estado de México. También está el patrimonio intangible que representan las fiestas religiosas, como la del pueblo de Huamantla, Tlaxcala.

En los libros de historia, poco se habla sobre la llamada guerra cristera que sucedió a fines de los años veinte y principios de los treinta del siglo XX; un conflicto 
armado que confrontó al gobierno con la institución católica. Ahora, en la zona donde el conflicto fue más fuerte, varios pueblos mágicos del estado de Guanajuato proponen desarrollar la ruta de la cristiada.

El programa Pueblos Mágicos también ha incentivado proyectos emprendedores. Ha habido cuestiones positivas en términos de creatividad, innovación impulso y entusiasmo por nuevos proyectos. La iniciativa promueve una actitud en la cual la gente toma en sus manos las alternativas económicas. Sin embargo, el problema que hemos registrado es la falta de continuidad de los mismos. Dos casos sirvan de ejemplo. En Bacalar, Quintana Roo, un grupo de norteamericanos hicieron paseos en barco y escenificaciones bajo el tema de los piratas, en tiempos en que estaban de moda las películas de Los Piratas del Caribe. Era un grupo que venía de California y se había entrenado como dobles de actuación. Estas personas estuvieron un tiempo en el pueblo mágico y luego regresaron a su país, dejando recuerdos y anécdotas.

Otro caso es el de unos jóvenes que en el pueblo de Huasca de Ocampo realizaban unos recorridos por la localidad, mientras contaban y escenificaban leyendas. A la iniciativa entusiasta, le siguieron problemas de trabajo en equipo y el grupo terminó por disolverse, lo que nos lleva a afirmar que más importante que las capacitaciones y las certificaciones para atender al turista, se necesitan cursos para apoyar proyectos emprendedores donde se les enseñe el manejo de conflictos, la planeación de una iniciativa y las formas de financiamiento.

Los casos mencionados dan cuenta de algunas dinámicas territoriales en los pueblos mágicos. Se trata de experiencias tan diversas como pueblos existen. Sin embargo, hay ciertas constantes, como la estandarización de la oferta turística bajo una especie de franquicia, una marca que tiene su propio logo que se repite hasta el cansancio por todo el país. Con ello, se reduce la riqueza cultural a las formas y fisonomías que pueden convertirse en mercancía.

\section{Conclusión}

El análisis de las territorialidades producidas a partir de la implementación del programa Pueblos Mágicos da cuenta de repercusiones en los ámbitos económico, cultural y político que van más allá del sector turismo, y que hacen patente la estructura de un sistema capitalista neoliberal.

En términos generales, creo que si bien la alternativa turística se presenta como una oportunidad ante la falta de empleo o los despojos territoriales por parte de ciertos sectores de la economía (como las mineras), el camino es complejo. Mal llevado puede incrementar el deterioro ambiental y orientar el patrimonio hacia las dinámicas del consumo en lugar de ser un baluarte de la memoria y la identidad cultural.

Las territorialidades dominantes registradas son las de las élites, los grupos en el poder que aprovechan bien el programa y desplazan los intereses de muchos pobladores locales. Al igual que en otras estructuras del sistema capitalista hay una concentración del poder político y económico. Y el panorama a futuro apunta hacia un 
deterioro ecológico y cultural, como el que vino como consecuencia de la industrialización.

Para un mejor funcionamiento el programa, considero que el turismo no debiera ser la única actividad económica presente en el ámbito local, que debe integrarse en un sistema, donde se complemente con otros sectores. Asimismo, es necesario retomar una visión territorial para buscar el beneficio de la comunidad, más que de ciertos grupos sociales.

De la investigación sobre el programa destaca la oposición existente entre el entusiasmo de los operadores turísticos y los funcionarios públicos frente a la exclusión y segregación de la población que no se siente parte del programa. Si bien es necesario abrir alternativas económicas en el marco de un país que está agotando sus recursos naturales y debe enfrentar una crisis social, económica y política, donde el despojo territorial dominante viene de las mineras, la violencia y los grandes proyectos inmobiliarios, considero que no se debe mirar únicamente al turismo, sin considerar un proyecto integral que incluya y le de importancia a otras actividades económicas que configuren un sistema territorial incluyente, ya que aunque en el discurso se enuncia que los habitantes deben ser considerados, deben participar y ser los beneficiarios de turistificación del lugar, la investigación realizada puede dar cuenta de procesos de despojo, exclusión, segregación y deterioro.

\section{Referencias}

ADORNO, T.; HORKHEIMER, M. Dialéctica de la ilustración. Madrid. Editorial Trotta. 2006.

ALVARADO, C. et al. Pueblos Mágicos. Narrativas varias. México. Pearson, Universidad Autónoma del Estado de Morelos, Universidad de Sonora. 2016.

CASTELLANOS A.; MACHUCA, J. A. Turismo, identidades y exclusión. México. Juan Pablos/ Universidad Autónoma Metropolitana Iztapalapa. 256p. 2008.

CONSEJO DE PROMOCION TURISTICA DE MEXICO. Panorama del sector turístico Disponible en: <http://www.cptm.com.mx/panorama-del-sectorturistico?language=es $>$ Consultado el 12 de enero de 2017.

DALTABUIT, M.; CISNEROS, H.; VALENZUELA, E. Globalización y sustentabilidad: el turismo en el sur de Quintana Roo. Cuernavaca. Centro Regional de Investigaciones Multidisciplinarias. UNAM. 2007.

DUARTE, E.; CUITZEO, M. La desapropiación social del patrimonio y el espacio público en: LÓPEZ LEVI, Liliana; VALVERDE VALVERDE, M. C. (Org.); FERNÁNDEZ PONCELA, A. M. (Org.); FIGUEROA, M. E. (Org.) Pueblos Mágicos: una visión interdisciplinaria. Volumen I. Ciudad de México. Universidad Autónoma Metropolitana y Universidad Nacional Autónoma de México. 2015. Págs. 159-185.

GIMENEZ, G. Territorio, cultura e identidades. La región socio-cultural en: Estudios sobre las culturas contemporáneas. Época II, vol V, num 9. p. 25-57. 1999. 
GONZÁLEZ TORREROS, L. Modelo turístico sustentable para el municipio de Tequila, Jalisco, México: una perspectiva del desarrollo local. Tesis de doctor en ciudad, territorio y sustentabilidad. Universidad de Guadalajara. Centro universitario de arte, arquitectura y diseño. 2010.

HAESBERT, R. El mito de la desterritorialización. México. Siglo XXI Editores. 2011.

HAESBERT, R. Del mito de la desterritorialización a la multiterritorialidad Cultura y representaciones sociales. Año 8, núm. 15, pg. 9 a 42, Septiembre 2013.

HERNÁNDEZ, J. Tequila: centro mágico, pueblo tradicional. ¿patrimonialización o privatización? en Andamios. Volumen 6, número 12, diciembre, 2009. p. 41-67.

HERNÁNDEZ LÓPEZ, J.; HERNÁNDEZ LÓPEZ, E. M. El paisaje agavero, patrimonio cultural de la humanidad: Una construcción política del paisaje y del patrimonio en: HERNÁNDEZ, J. J. (Org.); ROTMAN, M. B. (Org.); GONZÁLEZ, A. N. (Org.). Patrimonio y cultura en América Latina: Nuevas vinculaciones con el estado, el mercado y el turismo y sus perspectivas actuales. Guadalajara: Universidad de Guadalajara, 2010.

HERNÁNDEZ, M. (Org.) Pueblos mágicos: discursos y realidades. Universidad Autónoma Metropolitana. Ciudad de México. Unidad Lerma y Juan Pablos Editor. 2015.

LÓPEZ LEVI, L. (Org.); VALVERDE VALVERDE, M. (Org.); FERNÁNDEZ PONCELA, A. (Org.); FIGUEROA, M. (Org.) Pueblos Mágicos: una visión interdisciplinaria. Volumen I. Ciudad de México. Universidad Autónoma Metropolitana y Universidad Nacional Autónoma de México. 2015.

LÓPEZ LEVI, L. (Org.); VALVERDE VALVERDE, M. (Org.); FIGUEROA, M. (Org.) Pueblos Mágicos: una visión interdisciplinaria. Volumen II. Ciudad de México. Universidad Autónoma Metropolitana y Universidad Nacional Autónoma de México. 2015. LÓPEZ LEVI, L. (Org.); VALVERDE VALVERDE, M. (Org.); FIGUEROA, M. (Org.) Pueblos Mágicos: una visión interdisciplinaria. Volumen III. Ciudad de México. Universidad Autónoma Metropolitana y Universidad Nacional Autónoma de México. 2017.

MENDEZ, E. (Org.). Pueblos Mágicos. Variaciones de tradición y modernidad. Guadalajara. Universidad de Guadalajara. Laboratorio Nacional de Vivienda y Comunidades Sustentables. 2016.

MEXICO. GOBIERNO DE LA REPÚBLICA. Plan Nacional de desarrollo 20132018. México. Disponible en:

$<$ http://www.cmic.org.mx/comisiones/Sectoriales/turismo/2015/DOC_VIG_2015/ progrma_turismo.pdf>

OMT. Panorama OMT del turismo internacional. Organización mundial de turismo. 2016. Disponible en: <http://www.e-unwto.org/doi/pdf/10.18111/9789284418152>

OMT. Por qué el turismo. Disponible en: <http://www.eunwto.org/doi/pdf/10.18111/9789284418152>. Consultado el 23 de febrero de 2017. 
RAMÍREZ, I., OSORIO, M., NIETO, C., \& CORTÉS, I. “¿Así son, así se imaginan ellos, o así los imaginamos? Reflexiones sobre las transformaciones socioterritoriales del turismo residencial en Malinalco, México." Revista EURE - Revista De Estudios Urbano Regionales, 43(129). 2017

RODRÍGUEZ WALLENIUS C. Geopolítica del desarrollo local. México. Universidad Autónoma Metropolitana. Unidad Xochimilco. 2015.

SECTUR. Programa Pueblos Mágicos. México. Secretaría de Turismo. 2012

Recuperado el 26 de marzo de 2015 de

$<$ http://www.sectur.gob.mx/wb2/sectur/sect_Pueblos_Magicos>

SECTUR (s/f) Pueblos Mágicos. Reglas de operación. México. Secretaría de Turismo. Recuperado el 24 de junio de 2014 de

<http://www.sectur.gob.mx/work/models/sectur/Resource/99fbd793-a344-4b98-963378607f33cb8f/Reglas_de_operacion.pdf $>$

SECTUR Turismo, Nueva plataforma para el desarrollo social y económico: De la Madrid Comunicado 25. Disponible en: http://www.gob.mx/sectur/prensa/turismonueva-plataforma-para-el-desarrollo-social-y-economico-de-la-madrid

SECTUR Crece el PIB turístico más del doble que la economía nacional en el tercer trimestre de 2016. Disponible en: <http://www.gob.mx/sectur/prensa/crece-el-pibturistico-mas-del-doble-que-la-economia-nacional-en-el-tercer-trimestre-de-2016>

ALFONSO, V.; ALVARADO, C.; SALDAÑA, C.; GAMA, G. Imaginarios del paisaje y el turismo. Entre la tradición y distintivos oficiales. Ciudad de México. Juan Pablos Editor y Universidad Autónoma del Estado de Morelos. 2014.

\footnotetext{
Liliana López Levi

Doctora, maestra y licenciada en geografía por la Universidad Nacional Autónoma de México. Actualmente Profesora investigadora del Departamento de Política y Cultura de la Universidad Autónoma Metropolitana. Unidad Xochimilco, donde también es responsable de la especialidad de Sociedad y Territorio del Doctorado en Ciencias Sociales. Fue la Responsable Técnica del proyecto de investigación de ciencia básica "Los imaginarios del turismo: el caso de los Pueblos Mágicos". Proyecto que se desarrolló con el apoyo del Consejo Nacional de Ciencia y Tecnología (CONACYT).

Departamento de Política y Cultura. Calzada del Hueso 1100. Colonia Villa Quietud. Coyoacán 04960. Ciudad de México.

E-mail: levi_lili@yahoo.com.mx
}

Recebido para publicação em março de 2017 Aprovado para publicação em junho de 2017 